\title{
TESTING THE PERFORMANCE OF THE POWER LAW PROCESS MODEL CONSIDERING THE USE OF REGRESSION ESTIMATION APPROACH
}

\author{
Lutfiah Ismail Al turk \\ Statistics Department, King Abdulaziz University, Kingdom of Saudi Arabia
}

\begin{abstract}
Within the class of non-homogeneous Poisson process (NHPP) models and as a result of the simplicity of the mathematical computations of the Power Law Process (PLP) model and the attractive physical explanation of its parameters, this model has found considerable attention in repairable systems literature. In this article, we conduct the investigation of new estimation approach, the regression estimation procedure, on the performance of the parametric PLP model. The regression approach for estimating the unknown parameters of the PLP model through the mean time between failure ( $\mu T B F)$ function is evaluated against the maximum likelihood estimation (MLE) approach. The results from the regression and MLE approaches are compared based on three error evaluation criteria in terms of parameter estimation and its precision, the numerical application shows the effectiveness of the regression estimation approach at enhancing the predictive accuracy of the $\mu T B F$ measure.
\end{abstract}

\section{KEYWORDS:}

The Power Law Process Model,

Regression Estimation Approach, Maximum Likelihood Estimation, Time-Interval between Failures, Mean Time between Failures.

\section{INTRODUCTION}

The Power Law Process (PLP) model is a popular infinite NHPP model used to describe the reliability of repairable systems based on the analysis of observed failure data, this model was derived from the hardware reliability area. There is a lot of literature on the PLP model from a classical statistics view. Duane [1] was the first to propose the PLP model. During the development process of several failure systems, he presented failure data. Also, he formulated a mathematical relationship for predicting and observing the reliability enhancement as a function of cumulative failure time. He conducted several hardware applications in which the rate of failure occurrence was in a power law form in operating time. During the analysis of the failure data, it was noticed that the cumulative $\mu \mathrm{TBF}$ versus cumulative operating time followed a straight line on a log-log plot. After that, this model, comprehensively studied by Crow [2], he formulated the corresponding model as a non-homogeneous Poisson process (NHPP) with a power intensity law. 
The PLP model can be applied in software reliability although some problems may arises, it has been used in many successful applications, particularly in the defense industry. To model the failure rate of repairable systems, the PLP model is adopted by the United States Army Materials System Analysis Activity and called the Crow-AMSAA model. In standard applications it is assumed that the recurrence rate is the same for all systems that are observed. Bain [3] analyzed independent equivalent multi-system by employing the PLP model. Much theoretical work describing the PLP model was performed (examples; Lee, L. and Lee, K. [4], and Engelhardt and Bain [5]).

The PLP model has been widely used in reliability growth, in repairable systems, and software reliability models (see; Crow [6], Ascher and Feingold [7], and Kyparisis and Singpurwalla [8]). Littlewood [9] modified this model to overcome a problem associated with it, the problem of infinite failure rate at time zero. Rigdon [10] showed the wrongness of the claim that a linear Duane plot implies a PLP, and the conversion is false too.

In Calabria [11], the modified maximum likelihood (ML) estimators for the PLP model and the failure intensity of the expected number of failures in a given time interval is studied. Classical inference on the PLP model, such as point estimation, confidence intervals, tests of hypothesis for parameters and estimates of the intensity function was reviewed by Rigdon and Basu [12]. Yu et al. [13] considered the maximum likelihood estimates and the confidence intervals for the unknown parameters with left-truncated data. They also discussed the hypothesis testing and the goodness-of-fit test, and the predicted limits of future failure times for failure-truncated data.

Bayesian inference on the PLP model was also studied during the past two decades. One of the papers on Bayesian NHPP models is by Higgins and Tsokos [14] and the underlying model is the PLP model. Bayesian point and interval estimates were obtained by Guida [15], and Kyparisis and Singpurwalla [8]. Calabria et al. [16] considered the lower bounds for a test of equality of trends in several independent power law processes. Huang and Bier [17] presented a natural conjugate prior for the PLP model. By using the Bayesian method, Tian et al. [18] developed estimation and prediction methods for the PLP model in the left-truncated case. They also obtained the Bayesian point and credible interval estimates for the parameters of interest.

The extended case of inverting the Fisher information matrix to quantify the precision of the parameter estimates for the PLP model is considered by (Dyck and Verdonck [19]) where the recurrence rate between the different systems may vary with known scaling factors. They showed that the standard error of the parameter estimates can be quantified using analytical method.

In this article three test methods are applied through a numerical application to evaluate the effectiveness of the regression estimation approach that based on the $\mu$ TBF function. For the four real failure data that we considered in this article, we have shown that the regression estimation approach works better than the maximum likelihood approach. The regression approach gives less error in terms of all the used measurement criteria compared to the maximum likelihood approach. The rest of this article is organized as follows. In Section 2, the mathematical formulas of the PLP model's characteristics are presented. Section 3 briefly discusses the estimation of the unknown parameters for the PLP model, considering only the case of time-interval between failures. Section 4 presents briefly the selected evaluation criteria. Section 5 is an application to 
make a comparison between the performance of the regression estimation procedure and the method of likelihood estimation to four real data sets. Section 6 gives conclusion of data analysis.

\section{THE POWER LAW PROCESS (PLP) MODEL}

One particular and most commonly used model of the NHPP in the literature is the PLP, also called Duane, Crow-AMSAA, or Weibull process model. This model was first proposed by Duane [1] and later developed by Crow [2] as a NHPP model for hardware reliability. This model has the ability to be applied for the prediction of software reliability as well, and represents a functional relationship between two quantities, where one quantity varies as a power of another. With this model we can not make any relation between its parameters and the physical characteristics of the failure system. This model describes the failure history of reliable systems by supposing an NHPP for the counting process $\left\{\mathrm{N}\left(\mathrm{t}_{\mathrm{i}}\right), \mathrm{t}_{\mathrm{i}} \geq 0\right\}$, some of measures of reliability of the PLP model are: the mean value function or the expected cumulative number of failures in [0; $t$ ) and the conditional reliability function, the mathematical formulas of those measures are respectively as follows

$$
\mu\left(\mathrm{t}_{\mathrm{i}} ; \alpha, \eta\right)=\alpha t_{i}^{\eta}
$$

and

$$
\begin{aligned}
R\left(x \mid \mathrm{t}_{\mathrm{i}}\right) & =\mathrm{e}^{-\left\{\mu\left(\mathrm{t}_{\mathrm{i}}+\mathrm{x} ; \alpha, \eta\right)-\mu\left(\mathrm{t}_{\mathrm{i}} ; \alpha, \eta\right)\right\}} \\
= & \mathrm{e}^{-\alpha\left\{\left(\mathrm{t}_{\mathrm{i}}+\mathrm{x}\right)^{\eta}-t_{i}^{\eta}\right\}}
\end{aligned}
$$

The error-detection rate per error has the following form

$$
\begin{aligned}
d\left(\mathrm{t}_{\mathrm{i}} ; \alpha, \eta\right) & =\frac{\xi\left(\mathrm{t}_{\mathrm{i}} ; \alpha, \eta\right)}{\mathrm{n}\left(\mathrm{t}_{\mathrm{i}} ; \alpha, \eta\right)} \\
= & \eta\left(1-t_{i}^{\eta}\right)^{-1} t_{i}^{\eta-1}
\end{aligned}
$$

While the corresponding number of remaining error is given by

$$
\begin{gathered}
\mathrm{n}\left(\mathrm{t}_{\mathrm{i}} ; \alpha, \eta\right)=\alpha-\mu\left(\mathrm{t}_{\mathrm{i}} ; \alpha, \eta\right) \\
=\alpha\left(1-t_{i}^{\eta}\right)
\end{gathered}
$$

The failure intensity function depends only on the cumulative failure time and not on the previous pattern of failure times, it can be obtained as follows

$$
\begin{aligned}
\xi\left(\mathrm{t}_{\mathrm{i}} ; \alpha, \eta\right) & =\frac{\mathrm{d} \mu\left(\mathrm{t}_{\mathrm{i}} ; \alpha, \eta\right)}{\mathrm{dt}} \\
& =\alpha \eta t_{i}^{\eta-1}
\end{aligned}
$$

The mean time between failures ( $\mu \mathrm{TBF}$ ) can be found by the inverse of the intensity function

$$
\begin{aligned}
\mu \operatorname{TBF}\left(\mathrm{t}_{\mathrm{i}} ; \alpha, \eta\right) & =\frac{1}{\xi\left(\mathrm{t}_{\mathrm{i}} ; \alpha, \eta\right)} \\
= & (\alpha \eta)^{-1} t_{i}^{\eta-1},
\end{aligned}
$$


where $t_{i}>0, i=(1,2, \ldots, n)$ denote the failure times, $\alpha, \eta>0, \alpha$ is the scale parameter, and $\eta$ is the shape parameter. For the PLP model, if $\eta>1$, the failure intensity is increasing exponentially with time, the times between failures tend to be shorter, and this indicates that the software reliability is deteriorating. While if $\eta<1$, the failure intensity decreases sharply with time, the times between failures tend to be longer, then the software reliability of a system is being modeling with rapid improvement, and when $\eta=1$, the mean time between failures is equal to a constant value, the system is remaining stable over time and the PLP model reduces to the homogeneous Poisson process (HPP), [see; Figure 1].

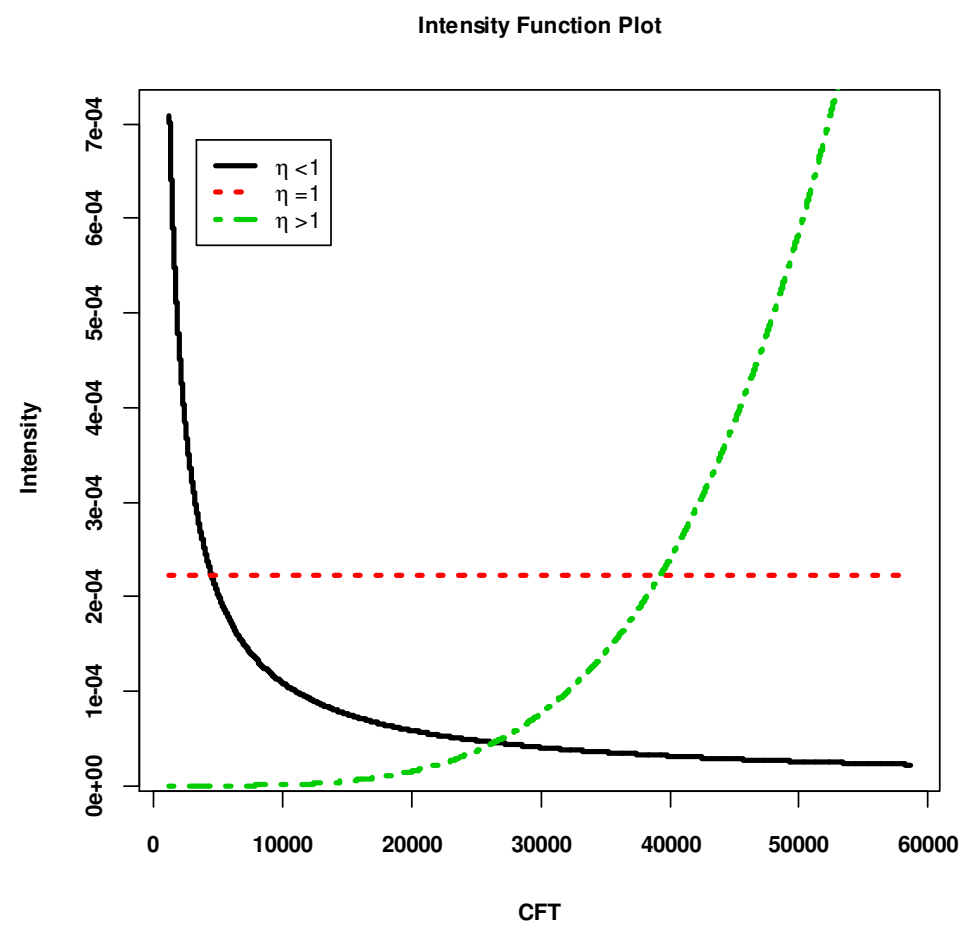

Figure 1. Intensity function plot of the PLP model.

\section{Estimation of the PLP Model's Parameters for the Case of Time Data}

Statistical inference procedures can be used easily and applied to the PLP model. For nonhomogeneous Poisson process (NHPP) models, there are two statistical categories, namely:

i. Time-interval between failures.

ii. Number of failures observed in a specified interval.

In this article the method of estimation will be applied to the first category, where successive times to failures are considered as a random variable. 


\subsection{The MLE approach of the PLP model}

It is easy to work out for estimation of the parameters for the PLP model, by considering equations (1) and (5) the corresponding cumulative mean value and intensity functions are respectively

$$
\left\{\begin{array}{l}
\mu\left(s_{i} ; \alpha, \eta\right)=\alpha s_{i}^{\eta} \\
\xi\left(s_{i} ; \alpha, \eta\right)=\alpha \eta s_{i}^{\eta-1}
\end{array}\right.
$$

Derived from the NHPPs and supposing that the cumulative failure time data $s_{i}(i=1,2, \ldots, n)$ are observed during the testing phase, then the likelihood function of the PLP model is defined as follows

$$
\begin{aligned}
\mathrm{L}(\alpha, \eta \mid \underline{S}) & =\mathrm{e}^{-\mu\left(s_{n} ; \alpha, \eta\right)} \prod_{i=1}^{\mathrm{n}} \xi\left(s_{i} ; \alpha, \eta\right) \\
& =\alpha^{\mathrm{n}} \eta^{\mathrm{n}} \mathrm{e}^{-\alpha \mathrm{s}_{\mathrm{n}}^{\eta}} \prod_{\mathrm{i}=1}^{\mathrm{n}} \mathrm{s}_{\mathrm{i}}^{\eta-1}
\end{aligned}
$$

And the natural logarithm of the joint density is obtained as

$$
\ln L(\alpha, \eta \mid \underline{s})=-\alpha s_{n}^{\eta}+n \ln \alpha+n \ln \eta+\sum_{i=1}^{n}(\eta-1) \ln s_{i}
$$

Proceed to estimate $\alpha$ and $\eta$. The first partial derivatives (with respect to $\alpha$ ) is taken and the equation is set equal to zero yield

$$
\frac{\partial \operatorname{lnL}(\alpha, \eta \mid \underline{\mathrm{s}})}{\partial \alpha}=-\mathrm{s}_{\mathrm{n}}^{\eta}+\frac{\mathrm{n}}{\alpha}=0
$$

Solving for $\alpha$, we have

$$
\hat{\alpha}_{\mathrm{MLE}}=\frac{\mathrm{n}}{\mathrm{s}_{\mathrm{n}}^{\eta}}
$$

Also, find the first partial derivatives (with respect to $\eta$ ) and Set the equation equal to zero yield

$$
\frac{\partial \ln L(\boldsymbol{\alpha}, \boldsymbol{\eta} \mid \underline{\mathrm{S}})}{\partial \boldsymbol{\eta}}=0
$$

giving

$s_{i}=\sum_{j=0}^{i} t_{j}, s_{n}=\sum_{j=0}^{n} t_{j}$

$$
\hat{\eta}_{M L E}=\frac{n}{n \ln s_{n}-\sum_{i=1}^{n} \ln s_{i}} \text {, where }
$$




\subsection{The regression estimation approach of the PLP model}

The $\mu \mathrm{TBF}$ is the basic measure of the system's reliability, for the regression procedure we use the $\mu \mathrm{TBF}$ to estimate the unknown parameters, by taking the natural logarithm of both sides of the $\mu \mathrm{TBF}$ function in Equation (6), we get

$$
\ln \left[\mu \mathrm{TBF}\left(\mathrm{s}_{\mathrm{i}}\right)\right]=-\ln (\alpha \eta)+(1-\eta) \ln \left(\mathrm{s}_{\mathrm{i}}\right)
$$

Using the method of regression estimation for the linear regression model, (Ryan [20]) we can derive the regression estimators of $\alpha$ and $\eta$ as follows

and

$$
\hat{\eta}_{\text {reg }}=1-\frac{\sum_{\mathrm{i}=1}^{\mathrm{n}} \ln \left(\mathrm{s}_{\mathrm{i}}\right) \ln \left(\mu \mathrm{TBF}\left(\mathrm{s}_{\mathrm{i}}\right)\right)-\frac{\sum_{\mathrm{i}=1}^{\mathrm{n}} \ln \left(\mathrm{s}_{\mathrm{i}}\right) \sum_{\mathrm{i}=1}^{\mathrm{n}} \ln \left(\mu \mathrm{TBF}\left(\mathrm{s}_{\mathrm{i}}\right)\right)}{\mathrm{n}}}{\sum\left[\ln \left(\mathrm{s}_{\mathrm{i}}\right)\right]^{2}-\frac{\left[\sum \ln \left(\mathrm{s}_{\mathrm{i}}\right)\right]^{2}}{\mathrm{n}}}
$$

$$
\hat{\alpha}_{\text {reg }}=\frac{e^{-\frac{\sum_{\mathrm{i}=1}^{\mathrm{n}} \ln \left(\mu \mathrm{TBF}\left(\mathrm{s}_{\mathrm{i}}\right)\right)}{\mathrm{n}}+\left(1-\hat{\eta}_{\mathrm{reg}}\right) \frac{\sum_{\mathrm{i}=1}^{\mathrm{n}} \ln \left(\mathrm{s}_{\mathrm{i}}\right)}{\mathrm{n}}}}{\hat{\eta}_{\mathrm{reg}}}
$$

Where $s_{i}, i=(1,2, \ldots, n)$ denote the cumulative failure times of occurrence,

\section{Error Measurement Criteria}

After the estimation of the model parameters, several tests exist to evaluate the performance of software reliability models quantitatively. The literature (Sharma et al. [21], Norman [22]) refers to several common evaluation criteria for software reliability models. In this section three of them are considered in order to evaluate the accuracy of the estimates from the PLP model, they are described below. Among them, the model performance is better when they are smaller.

\subsection{The Bias}

The bias is the sum of the difference between the estimated curve and the actual data, and computed by

$$
\left.\operatorname{Bias}=\frac{1}{n} \sum_{i=1}^{n}(\mu \mathrm{TBF}(\mathrm{t} ; \alpha, \eta)-\mu \mathrm{TB} \widehat{\mathrm{F}(\mathrm{t} ;} \alpha, \eta)\right)
$$

\subsection{The mean squared error (MSE)}

The MSE is the most widely used evaluation criteria of prediction. It is used to describe the deviation between the predicted values with the actual values, and is defined as

$$
\left.\operatorname{MSE}=\frac{1}{n-p} \sum_{i=1}^{n}(\mu \operatorname{TBF}(\mathrm{t} ; \alpha, \eta)-\mu \mathrm{TB} \widehat{\mathrm{F}(\mathrm{t} ;} \alpha, \eta)\right)
$$


International Journal of Software Engineering \& Applications (IJSEA), Vol.5, No.5, September 2014

\subsection{The mean error of prediction (MEOP)}

The MEOP is the summation of the absolute value of the variation between the real data and the predicted values. The equation of evaluating the MEOP is given by

$$
\left.\mathrm{MEOP}=\frac{1}{n-p+1} \sum_{i=1}^{n} \mid \mu \mathrm{TBF}(\mathrm{t} ; \alpha, \eta)-\mu \mathrm{TB} \widehat{\mathrm{F}(\mathrm{t} ; \alpha}, \eta\right) \mid,
$$

where $\mathrm{n}$ represents the number of measurements used for estimating the model parameters, and $\mathrm{p}$ the number of parameters.

\section{Empirical Application}

In our analysis, four real failure data sets are analyzed using the PLP model, to easy implement the analysis of failure data, $\mathrm{R}$ code is created. The first step and for the case of TBF data, the estimates of the unknown parameters are found using the MLE and the regression approaches. Both objective and graphical method based on the cumulative mean time between failures are used to study the effectiveness of the regression estimation procedure. Three evaluation criteria are used to evaluate the prediction results.

\subsection{Failure datasets}

For evaluation the performance of the regression estimation approach with the traditional MLE approach, four actual software reliability failures data are analyzed. The first data set is presented by Musa [23]. The second data set is taken from Jeske [24].The Apollo 8 software test data is first reported in Jelenski and Moranda [25]. Tohma's software failure data is reported and studied by Tohma [26]. Figure 2 shows the time between failures versus the failure number for all the selected failure data. 


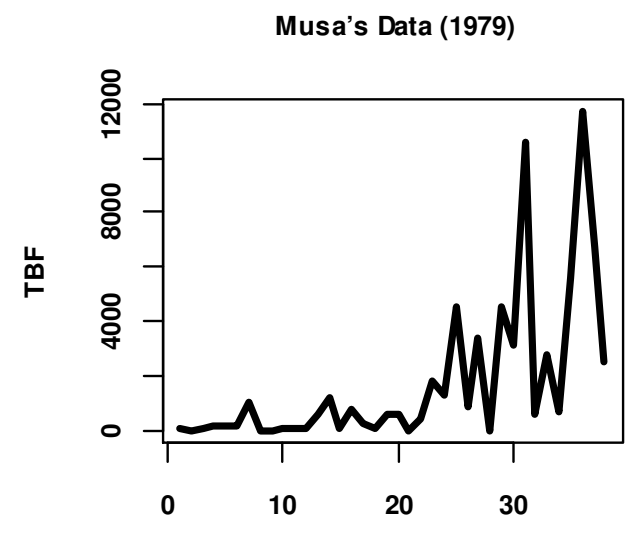

Failure Number

Apollo 8 Data (1972)

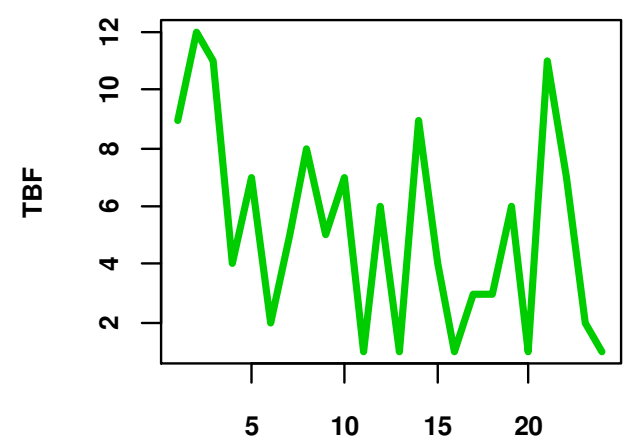

Failure Number

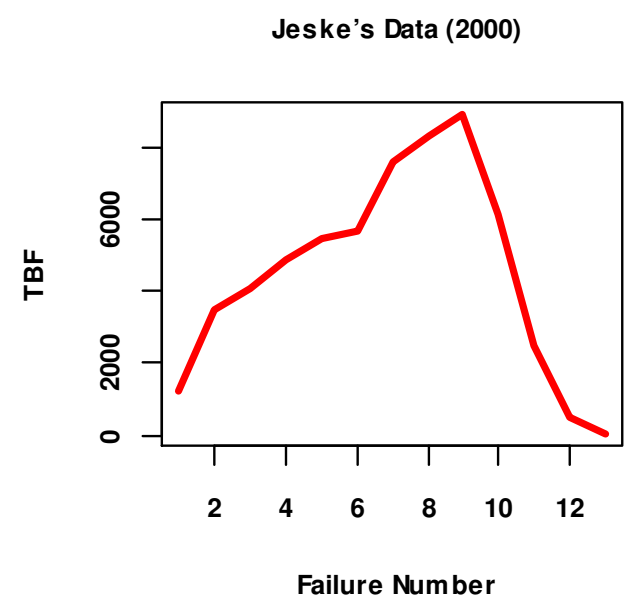

Tohma's Data (1989)

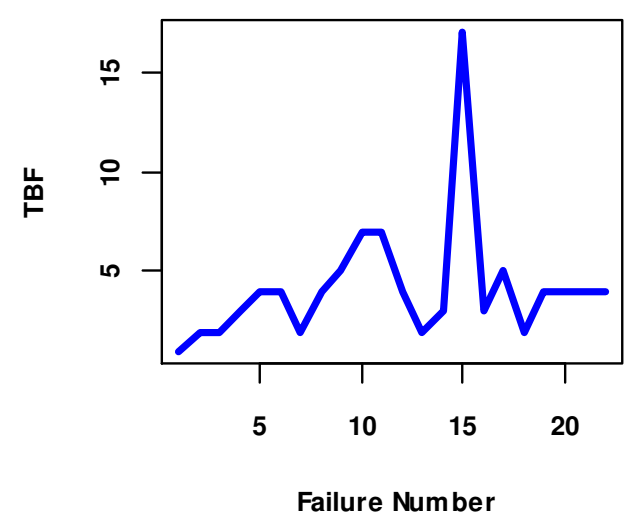

Figure 2. The time between failures versus the failure number

\subsection{Discussion}

To test the performance of the PLP model for the four selected failure data, first the estimates of the unknown parameters $\alpha$ and $\eta$ are obtained using the MLE and the regression approaches. For the regression the reliability metric $\mu \mathrm{TBF}$ is considered. The actual and the predicted cumulative $\mu \mathrm{TBF}$ using the two method of estimation is represented graphically in Figure 3. From this figure we can see that, according to the $\mu \mathrm{TBF}$ regression approach, it appears that the PLP model estimates the $\mu \mathrm{TBF}$ very well for the four selected failure data sets, the predictive $\mu \mathrm{TBF}$ curve is very closely related to the actual $\mu \mathrm{TBF}$ curve which suggests that the four selected failure data sets do follow the PLP model very closely. But this is not the case when using the MLE approach, as this parametric approach gives poor prediction results. In Table 1 the MSE, Bias, and MEOP is reported, the obtained lower values of this measures considering the regression estimation 
International Journal of Software Engineering \& Applications (IJSEA), Vol.5, No.5, September 2014

approach indicates the effective capability of using this procedure. All the selected performance measures agree on that the regression approach gives an enhanced prediction results.

Figure 3.a Musa's Data (1979)

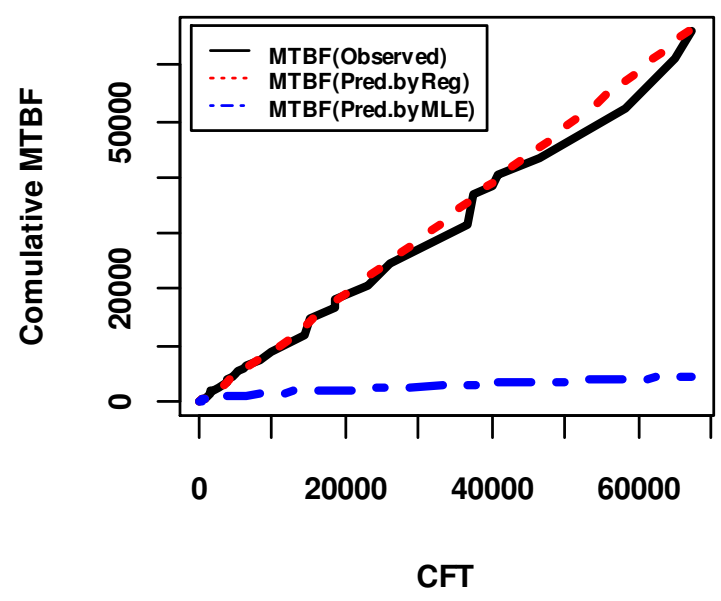

Figure 3.c Apollo 8 Data (1972)

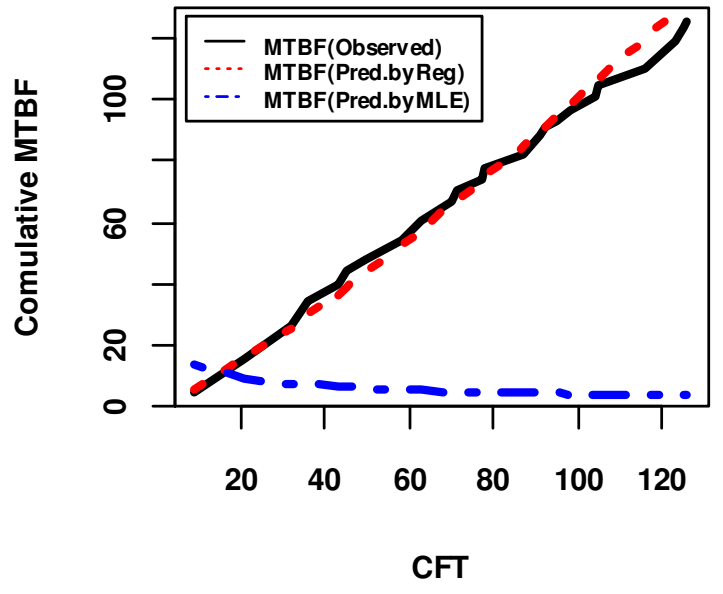

Figure 3.b Jeske's Data (2000)

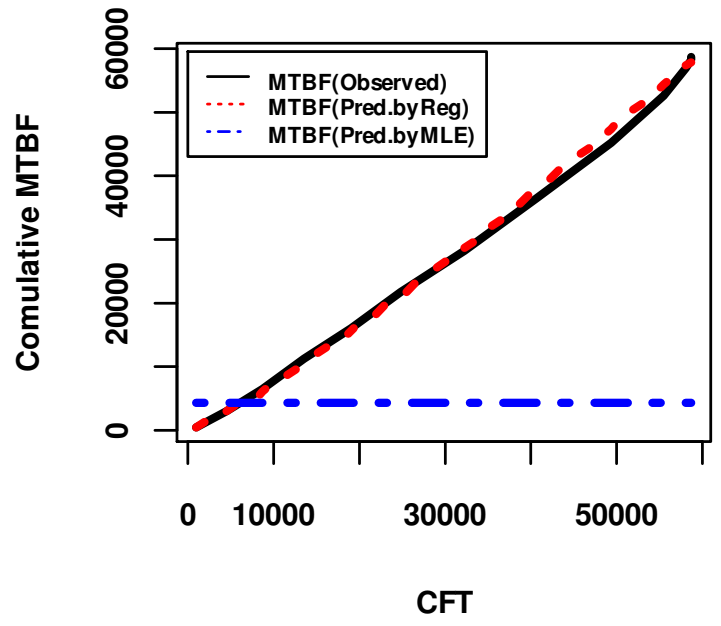

Figure 3.d Tohma's Data (1989)

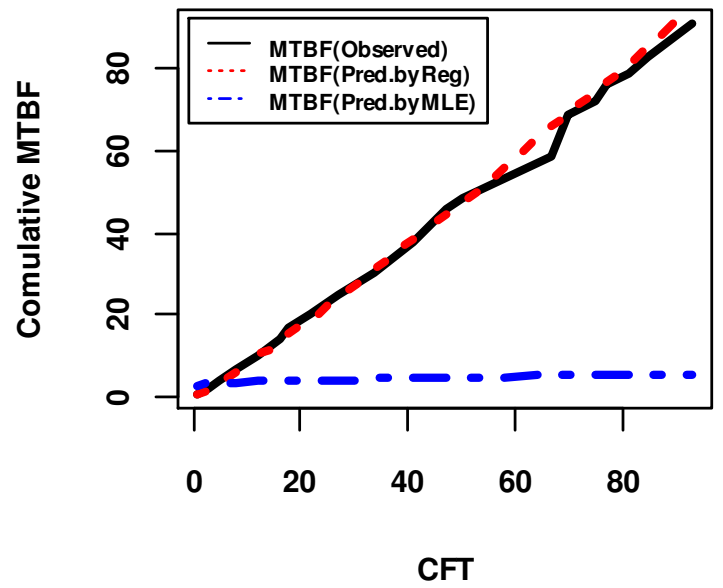

Figure 3. Cumulative $\mu \mathrm{TBF}$ vs cumulative failure time plot 
International Journal of Software Engineering \& Applications (IJSEA), Vol.5, No.5, September 2014

Table 1. The MSE, Bias, and MEOP of the two estimation approaches

\begin{tabular}{|l|c|c|c|}
\hline Datsets & Evaluation & MLE & Regression \\
& criteria & approach & approach \\
\hline Musa's Data (1979) & MSE & 483927928 & 1739304 \\
\cline { 3 - 4 } & Bias & 13067.4700 & 348.8833 \\
\cline { 3 - 4 } & MEOP & 13420.6500 & 358.3126 \\
\hline Jeske's Data (2000) & MSE & 1330498442 & 1263012 \\
\cline { 3 - 4 } & Bias & 25966.6200 & 219.7441 \\
\cline { 3 - 4 } & MEOP & 28130.5000 & 238.0561 \\
\hline Apollo 8 Data (1972) & MSE & 6402.7620 & 21.2888 \\
\cline { 3 - 4 } & Bias & 67.8297 & 0.8530 \\
\cline { 3 - 4 } & MEOP & 70.7788 & 0.8901 \\
\hline Tohma's Data (1989) & MSE & 2505.0440 & 5.4390 \\
\cline { 3 - 4 } & Bias & 37.5658 & 0.6771 \\
\cline { 3 - 4 } & MEOP & 39.3546 & 0.7093 \\
\hline
\end{tabular}

\section{CONCLUSION}

One of the earliest proposed and common used reliability models is the PLP model, it is simple flexible and has been doing very well in many applications for reliability prediction. It can be used to model deteriorating systems as well as to model developing systems. In this article, the performance of the PLP model has been evaluated for several repairable data based on the MLE method which can be obtained using the likelihood function, and the regression estimation approach based on the $\mu \mathrm{TBF}$ function of cumulative failure time data. According to the selected error measurement criteria, the use of the regression estimation approach resulted in muchenhanced predictive capability, it produces good evaluation of the $\mu \mathrm{TBF}$. The MLE approach gives poor results in terms of three different evaluation criteria. This suggests that, in software reliability modeling, the first step should be to consider the regression approach for software reliability prediction.

\section{REFERENCES}

[1] Duane, J. T. (1964). Learning Curve Approach to Reliability Monitoring, IEEE Trans. Aerospace Electron System, 2, 563-566.

[2] Crow, L. H. (1974). Reliability for Complex Systems, Reliability and Biometry. Society for Industrial and Applied Mathematics (SIAM), pp. 379-410.

[3] Bain, L. J. (1978). Statistical Analysis of Reliability and Life Testing Models, Decker, New York.

[4] Lee, L. and Lee, K. (1978). Some Results on Inference for the Weibull Process, Technometrics, 20, 41-45.

[5] Engelhardt, M. and Bain, L. J. (1986). On the Mean Time Between Failures for Repairable Systems. IEEE Transactions on Reliability, R-35, 419-422.

[6] Crow, L. H. (1982). Confidence Interval Procedures for the Weibull Process with Applications to Reliability Growth. Technometrics, 24, 67-72.

[7] Ascher, H. and Feingold, H. .(1984). Repairable Systems Reliability, Decker, New York. 
International Journal of Software Engineering \& Applications (IJSEA), Vol.5, No.5, September 2014

[8] Kyparisis, J. and Singpurwalla, N. D. (1985). Bayesian Inference for the Weibull Process with Applications to Assessing Software Reliability Growth and predicting Software Failures. Computer Science and Statistics: Proceedings of the Sixteenth Symposium on the Interface (ed. L. Billard), 5764, Elsevier Science Publishers B. V. (North Holland), Amsterdam.

[9] Littlewood, B. (1984). "Rationale for modified Duame model", IEEE Trans. Reliability, Vol. R-33 No.2, pp.157-9.

[10] Rigdon, S. E. (2002). Properties of the Duane plot for repairable systems.Quality and Reliability Engineering International 18, 1-4.

[11] Calabria, R. Guida, M. and Pulcini, G. (1988). Some Modified Maximum Likelihood Estimators for the Weibull Process. Reliability Engineering and System Safety, 23, 51-58.

[12] Rigdon, S. E. and Basu, A. P. (1990). Estimating the Intensity Function of a Power Law Process at the Current Time: Time Truncated Case. Communications in Statistics Simulation and Computation, 19, 1079-1104.

[13] Yu, J. W., Tian, G. L. and Tang, M. L. (2008). Statistical inference and prediction for the Weibull process with incomplete observations. Computational Statistics and Data Analysis 52, 1587-1603.

[14] Higgins, J. J. and Tsokos, C. P. (1981). A Quasi-Bayes Estimate of the Failure Intensity of a Reliability Growth Model, IEEE Trans. on Reliability.

[15] Guida, M. , Calabria, R. and Pulcini, G. (1989). Bayes Inference for a Non-homogeneous Poisson Process with Power Law Intensity. IEEE Transactions on Reliability. R-38, 603-609.

[16] Calabria, R., Guida, M. and Pulcini, G. (1992). Power Bounds for a Test of Equality of Trends in k Independent Power Law Processes. Comm. Statist. Theory Methods, 21(11): 3275-3290.

[17] Huang,Y. and Bier,V. (1998). A Natural Conjugate Prior for the Non-Homogeneous Poisson Process with a Power Law Intensity Function, Commun. Statist. 27(2): 525-551.

[18] Guo-Liang Tian, Man-Lai Tang, and Jun-Wu Yu, (2011). Bayesian Estimation and Prediction for the Power Law Process with Left-Truncated Data. Journal of Data Science, 445-470.

[19] Van Dyck, Tim Verdonck, (2014). Precision of power-law NHPP estimates for multiple systems with known failure rate scaling. Rel. Eng. \& Sys. Safety 126: 143-152.

[20] Ryan S. E, and L. S. Porth, A Tutorial on the Piecewise Regression Approach Applied to Bedload Transport Data, Gen. Tech. Rep. RMRS-GTP-189. Fort Collins, CO. U. S. Department of Agriculture, Forest Service, Rocky Mountain Research Station, 2007.

[21] Kapil Sharma, Rakesh Garg, Nagpal C. K., and Garg R. K. (2010). "Selection of Optimal Software Reliability Growth Models Using a Distance Based Approach", IEEE Transactions On Reliability, Vol. 59, No. 2, Page 266-277.

[22] Norman F. Schneidewind. (1993). "Software Reliability Model with Optimal Selection of Failure Data", IEEE Transactions on Software Engineering, Vol.19, No. 11, Page 1095-1105.

[23] Musa, J. D. (1979). Software Reliability Data. Report Available from Data \& Analysis Center for software, Rome Air Development Center, Rome, New York, USA.

[24] Jeske, D. R. and Qureshi, M. (2000). Estimating the Failure Rate of Evolving Software, Proceedings of 11 th International Symposium on Software Reliability Engineering (San Jose) (Est. Acceptance Ratio: 40\%), pp. 52-61.

[25] Jelinski Z., and Moranda P. B. (1972). Software Reliability Research. Statistical Computer Performance Evaluation. Academic Press, NewYork, pp. 465-484.

[26] Tohma Y., Jacoby R., Murata Y., and Yamamoto, M. (1989). "Hyper-Geometric Distribution Model to Estimate the Number of Residual Software Faults," Proc. COMPsac-89, Orlando, pp. 610-617. 
International Journal of Software Engineering \& Applications (IJSEA), Vol.5, No.5, September 2014

\section{Authors}

Lutfiah Ismail Al turk is presently Assistant Professor of Mathematical Statistics in Statistics Department at Faculty of Sciences, King AbdulAziz University, Saudi Arabia. Lutfiah Ismail Al turk obtained her B.Sc degree in Statistics and Computer Science from Faculty of Sciences, King AbdulAziz University in 1993 and M.Sc (Mathematical statistics ) degree from Statistics Department, Faculty of Sciences, King AbdulAziz University in 1999. She received her Ph.D in Mathematical Statistics from university of Surrey, UK in 2007. 